\title{
Cost-effectiveness evaluation of glucosamine for osteoarthritis based on simulation of individual patient data obtained from aggregated data in published studies
}

\author{
Olivier Bruyère $^{1} \mathbb{D} \cdot$ Jean-Yves Reginster ${ }^{1,2} \cdot$ Germain Honvo $^{1} \cdot$ Johann Detilleux $^{1,3}$
}

Received: 26 November 2018 / Accepted: 18 January 2019 / Published online: 12 February 2019

(C) The Author(s) 2019

\begin{abstract}
Background The economic evaluation of treatments usually requires access to individual patient data, which is difficult to obtain. Moreover, in osteoarthritis, health utility scores are unavailable and can be assessed only using a validated equation model based on various clinical data. We aimed to develop and validate a methodology to simulate individual health utility scores from aggregated clinical data available in published studies to calculate the cost-effectiveness of different glucosamine preparations (i.e., crystalline glucosamine sulfate, glucosamine sulfate, and glucosamine hydrochloride) used for osteoarthritis.

Methods We developed a method to simulate individual utility values and validated the model by comparing the results obtained with the simulation and the results of one trial where the utility scores are available. Then, we simulated the utility scores of 10 published trials that used different glucosamine preparations. The utility estimates were used to calculate the quality-adjusted life year (QALY) using the area-under-the-curve method. Costs were for the glucosamine product only. The incremental cost/effectiveness ratio (ICER) was then calculated.

Results The values of utility scores calculated from data sources and those simulated with the model were similar. From 10 studies where utility was simulated, four used crystalline glucosamine sulfate, and six used other formulations. The ICER revealed that compared to placebo, crystalline glucosamine sulfate only was cost-effective at all time points and up to 3 years with a median ICER of 5347.2 €/QALY at month 3, 4807.2 €/QALY at month 6 and 11535.5 €/QALY at year 3. The use of other formulations was not cost-effective.

Conclusion Using a new model to simulate individual health utility scores of patients included in ten published trials, ICER analysis showed that the use of crystalline glucosamine sulfate is cost-effective, while other formulations were not. The results confirm the importance of the formulation of glucosamine products.
\end{abstract}

Keywords Osteoarthritis $\cdot$ Cost-effectiveness $\cdot$ Glucosamine

Olivier Bruyère

olivier.bruyere@uliege.be

1 Department of Public Health, Epidemiology and Health Economics, WHO Collaborating Centre for Public Health Aspects of Musculo-Skeletal Health and Ageing, University of Liège, 4000 Liège, Belgium

2 Prince Mutaib Chair for Biomarkers of Osteoporosis, Biochemistry Department, College of Science, King Saud University, Riyadh 11451, Saudi Arabia

3 Department of Veterinary Management of Animal Resources, University of Liège, Liège, Belgium

\section{Introduction}

Osteoarthritis (OA) is a major public health problem because of its current and future prevalence, its impact on mortality and morbidity and the associated healthcare cost [1]. Pharmacological and non-pharmacological treatments are currently available and their effects in reducing the symptoms of OA or increasing the quality of life of OA patients have been widely assessed by different scientific organisations [2-4]. A treatment algorithm for the management OA was also proposed, which provides practical guidance for the prioritisation of interventions [5]. In this algorithm, the use of symptomatic slow-acting drugs for osteoarthritis (SYSADOA) as the first pharmacological therapy is recommended. 
However, some international scientific societies do not recommend the use of SYSADOAS in their most recent guidelines $[2,4,6]$. Indeed, there are many different agents in the class of SYSADOAs, including glucosamine, chondroitin, diacerein, and avocado soybean unsaponifiables, and not all are supported with a high level of clinical efficacy data, nor supported by the same degree of recommendation in clinical guidelines. Although more studies are needed to further substantiate their precise effects, some evidence is available with the use of glucosamine and chondroitin as SYSADOAs with an impact on both symptoms and structure in the long term. Multiple formulations of glucosamine and chondroitin are available as both prescription-grade products and nutritional supplements, and these differences have been hypothesised as being an important driver of the discrepancy in the level of recommendation of SYSADOAS between guidelines [7]. Indeed, although all preparations may claim to have a therapeutic effect, not all are supported by clinical evidence [8]. For example, with glucosamine, an independent metaanalysis has shown that in trials using a specific formulation of glucosamine, the prescription crystalline glucosamine sulfate (pCGS) had a better outcome on pain than did other preparations of glucosamine [9].

In a world with limited resources and health care budgets, it is important to allocate scarce resources efficiently and consequently, to develop effective treatments and efficient strategies. Economic evaluation is a method for comparing different strategies in terms of cost (e.g., intervention costs and disease costs) and consequences [e.g., life years or quality-adjusted life year (QALY)]. These evaluations play a growing role in pricing and reimbursement decisions as regulatory agencies rely more and more on pharmacoeconomic data to make decisions about limited resources [10]. To the best of our knowledge, only one clinical trial has explored the cost-effectiveness of glucosamine (and in this particular case the pCGS formulation) compared with that of paracetamol and placebo in the treatment of knee OA [11]. The authors concluded that compared with paracetamol and placebo, pCGS was a highly cost-effective therapy to treat patients diagnosed with knee OA. However, another research group using a cohort simulation model based on clinical data available from five systematic reviews and one clinical guideline showed that there was evidence that glucosamine sulfate shows some clinical effectiveness in the treatment of $\mathrm{OA}$ of the knee [12]. However, in this economic evaluation the authors highlighted the need for further research since they showed that estimates were imprecise and subject to a degree of decision uncertainty.

To further advance the economic evaluation of glucosamine, we should theoretically have access to individual patient data. Unfortunately, most of the time, access to these data is not possible because of technical, legal or patient willingness issues. Consequently, we decided to develop and validate a method to simulate individual health utility scores based on the aggregated clinical data provided in published papers of clinical trials. Based on this method of simulation, we performed some cost-effectiveness analyses based on trials that used different formulations of glucosamine.

\section{Materials and methods}

\section{Development of the simulation model}

To simulate individual values, we had to solve two major issues. The first issue is that health utility scores are not directly assessed in most OA studies in contrast to WOMAC (i.e., an OA specific health-related quality of life questionnaire) scores. Interestingly, Grootendorst et al. [13] have developed a linear regression model to estimate utility scores based on the age of the patient, the number of years since he/ she was diagnosed with OA and the three different WOMAC subscales scores. The second issue is that only summary statistics (mean, median, standard deviation, covariance or confidence interval) of age, number of years since OA and WOMAC scores are reported in most OA trials.

To overcome these issues, we propose the following procedure to obtain individual health utility scores:

- Use the SIMNORMAL procedure of SAS and published summary statistics to simulate individual values for the WOMAC indexes, age and years since OA. The SIMNORMAL procedure performs conditional and unconditional simulation for a set of correlated normal or Gaussian random variables.

- Discard simulated values outside the following permissible ranges: $0-20$ for pain, $0-68$ for function, $0-8$ for stiffness, $0-100$ for age and $0-100$ for years since OA diagnosis.

- Compute individual female utility scores from the equation provided in the paper by Grootendorst (considering that the parameter "female" in the equation of Grootendorst takes the value of 1 if the patient is a woman).

To validate the procedure, we tested it on data from the only study on glucosamine in which individual health utility values were published [11] and for which we have access to the individual values for WOMAC scores, age and years since OA diagnosis, at baseline and after 3 months of treatment. We computed means and standard deviations for and between these values with the CORR procedure of SAS at baseline, after 3 months and for subjects in the placebo and pCGS groups. Next, we compared simulated and published values. 


\section{Simulation of individual health utility scores}

We used the procedure to simulate individual health utility scores for all clinical trials cited in the meta-analysis of Eriksen et al. [9] that have used the WOMAC. Consequently, we simulated individual health utility scores from ten trials (4 using pCGS and 6 using other forms of glucosamine) [14-23]. When available, means and SD were extracted from published articles, after correction for the scales (to be on the scale for WOMAC indexes as the one used in the equation of Grootendorst). We replaced missing data in the summary statistic of published studies (i.e., sometimes data, such as the standard deviation or a specific variable such as years since OA, were unavailable) with data from the study used to develop and validate the procedure [11]. We simulated a total of 20,000 patients in each study $(10,000$ glucosamine and 10,000 placebo), and data were examined by two independent experts.

\section{QALY assessment}

The utility estimates were used to calculate the QALY using the area-under-the-curve method [24] that is the weighted average of time spent in the study and utility value. If more than one study was available for a particular time (e.g., 3 months), we weighted each study according to the number of subjects included in the trial.

\section{Costs of glucosamine}

Since there are major differences in products, reimbursement strategies, daily dosage, among other factors, the range of costs of glucosamine products varies widely. To get an official overview, we could access the official IMS Health data, updated at December 2017, related to the main selling prices of the different formulations, in the different countries. Since the data is reported in both local currency and US dollars, it was possible to report data in US dollars and then in Euros with homogeneous currency exchange rate. Obviously, we separated pCGS and other forms of glucosamine. Since the price range was quite wide, to reduce variability and define a reasonably weighted average price for each of the two forms of glucosamine, the following methodology was devised: after calculation of the overall average price, all prices that were $>50 \%$ lower than this average price were excluded, and the new average of the remaining prices was calculated and defined as the "higher" value of the cost-range. In the same way, we started again with all prices and excluded those that were $>50 \%$ higher than the overall average, and calculated a new average of the remaining prices, which was defined as the "lower" value of the price range. Consequently, we achieved a median cost of $0.79 € /$ day for pCGS (with low and high prices of $0.65 € /$ day and $0.88 € /$ day, respectively) and a median cost of $0.55 € /$ day for other forms of glucosamine (with low and high prices of $0.45 € /$ day and $0.66 € /$ day, respectively). The price of placebo was considered $0 €$.

\section{Cost-effectiveness analysis}

The incremental cost/effectiveness ratio (ICER)—a measure of the additional cost per unit of health gain - was then calculated. The underlying calculation for the ICER comparing glucosamine products vs. placebo in patients with knee OA was equal to (average cost glucosamine - average cost placebo)/(average effect glucosamine - average effect placebo) where costs were measured in Euros, and effects were measured in QALY.

\section{Sensitivity analysis}

Because of the different time points used in different studies (i.e., from 2 months to 3 years), we decided to use data from longer studies at all time points. For example, for a 3 -year study, we considered an $8.3 \%$ of the global effect at month $3(3 / 36 \times 100=8.3 \%)$ and a $16.7 \%$ of the global effect at month $6(6 / 36 \times 100=16.7)$. Obviously, we acknowledge that this approach could reduce the potential (cost)-effectiveness of the treatment since it has been shown that at least 3 months is needed to begin seeing an effect with most of the SYSADOAs.

\section{Results}

From the study of Herrero-Beaumont et al. [16], the simulated mean health utility score was 0.598 at baseline and 0.676 at the end of the study in the glucosamine group. These values were respectively 0.602 and 0.651 in the placebo group. The change over time was 0.08 in the glucosamine group and 0.05 in the placebo group. Interestingly, these values are completely similar to the previously published results (i.e., 0.59 and 0.60 at baseline and a 0.08 and 0.05 change over time in the glucosamine group and the placebo group, respectively) suggesting that the simulation model provides reliable utility value [11].

The differences between the glucosamine and placebo groups in the changes over time of the simulated utility scores of all clinical trials that have used the WOMAC are presented in Table 1. As expected from previously published WOMAC results [9], the differences in changes in utility values were more often in favour of glucosamine in trials that used pCGS than in those that used other formulations.

The evolution of the health utility scores among studies that used pCGS or other forms of glucosamine is presented in Fig. 1. The utility score always improved when pCGS was 
Table 1 Health utility changes in the studies using pCGS or other forms of glucosamine

\begin{tabular}{|c|c|c|c|c|c|}
\hline & Type of glucosamine & $N$ & $\begin{array}{l}\text { Duration } \\
\text { (month) }\end{array}$ & $\begin{array}{l}\text { Health utility changes in the } \\
\text { glucosamine group }\end{array}$ & $\begin{array}{l}\text { Health utility changes } \\
\text { in the Placebo group }\end{array}$ \\
\hline Giordano et al. [17] & pCGS & 60 & 3 & 0.135 & -0.0742 \\
\hline Beaumont et al. [16] & pCGS & 210 & 6 & 0.0785 & 0.0315 \\
\hline Reginster et al. [14] & pCGS & 212 & 36 & 0.1606 & 0.1427 \\
\hline Pavelka et al. [15] & pCGS & 202 & 36 & 0.0487 & 0.0207 \\
\hline Houpt et al. [18] & Other form of glucosamine & 101 & 2 & 0.0293 & 0.0129 \\
\hline McAlindon et al. [19] & Other form of glucosamine & 205 & 3 & -0.0465 & -0.0339 \\
\hline Frestedt et al. [22] & Other form of glucosamine & 35 & 3 & 0.1629 & 0.0613 \\
\hline Chopra et al. [25] & Other form of glucosamine & 70 & 3 & 0.0016 & 0.1232 \\
\hline Cibere et al. [20] & Other form of glucosamine & 137 & 6 & 0.0096 & 0.0006 \\
\hline Clegg et al. [21] & Other form of glucosamine & 630 & 6 & 0.0242 & 0.0383 \\
\hline
\end{tabular}

Fig. 1 Health utility evolution in trials having used pCGS or other forms of glucosamine

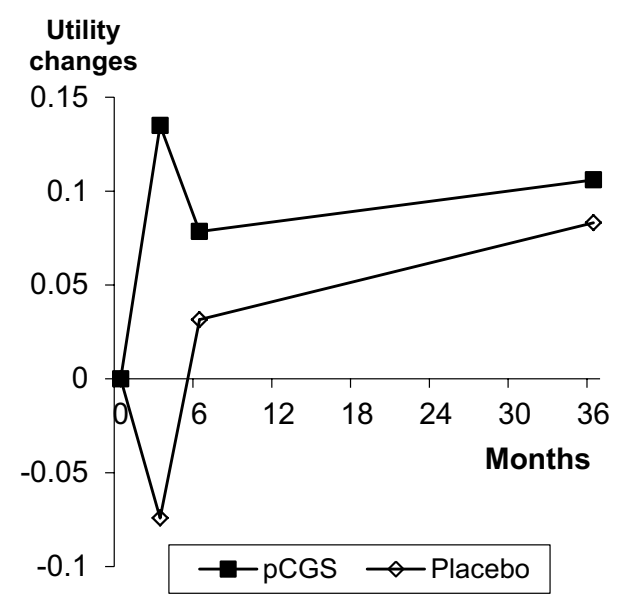

Table 2 Incremental cost-effectiveness ratio results of studies having used pCGS

\begin{tabular}{lccc}
\hline & At 3 months & At 6 months & At 36 months \\
\hline QALY change pCGS & 0.016875 & 0.0435625 & 0.27418931 \\
QALY change placebo & -0.009275 & -0.0146125 & 0.12872929 \\
Median cost pCGS & 139.83 & 279.66 & 1677.96 \\
Median ICER & 5347.2 & 4807.2 & 11535.5 \\
Lowest cost pCGS & 115.05 & 230.1 & 1380.6 \\
Lowest ICER & 4399.61759 & 3955.30726 & 9491.2675 \\
Highest cost pCGS & 155.76 & 311.52 & 1869.12 \\
Highest ICER & 5956.40535 & 5354.87752 & 12849.716 \\
\hline
\end{tabular}

used while the results are much more variable when other formulations of glucosamine were used.

The calculation of the ICER among studies having used pCGS show that the use of this formation is highly costeffective compared to placebo, regardless of the price of the treatment (Table 2). The ICER calculation also shows that pCGS is already cost-effective after 3 months of treatment and up to 3 years after the initiation of the treatment.
When looking at other forms of glucosamine, notably, if the ICER results could be considered cost-effective at 2 months (based on one single study), the results no longer show the cost-effectiveness of these other formulations of glucosamine at 3 months (Table 3). Moreover, at 6 months of treatment, from a health economics perspective, placebo is even better than these formulations. None of these results are substantially influenced by the costs of glucosamine.

The results of sensitivity analyses, using data from longer studies at all time points, are presented in Fig. 2 and Tables 4 and 5. For pCGS, the health utility change is always in favour of the treatment compared to placebo and the ICER shows a cost-effectiveness that increases over time. Using other formulations of glucosamine, no meaningful difference in utility scores is observed compared to placebo and the cost-effectiveness analyses confirm the absence of economic interest of these formations at all time points. 
Table 3 Incremental costeffectiveness ratio results of studies having used other forms of glucosamine

\begin{tabular}{llcl}
\hline & At 2 months & At 3 months & At 6 months \\
\hline QALY change other glucosamine & 0.002344 & 0.00303613 & 0.00423555 \\
QALY change placebo & 0.001032 & 0.0020409 & 0.00752699 \\
Median cost other glucosamine & 33 & 49.5 & 99 \\
Median ICER & $25,152.4$ & $49,737.4$ & Placebo better \\
Lowest cost other glucosamine & 27 & 40.5 & 81 \\
Lowest ICER & $20,579.2$ & $40,694.2$ & Placebo better \\
Higesth cost other glucosamine & 39.6 & 59.4 & 118.8 \\
Highest ICER & $30,182.9$ & $59,684.9$ & Placebo better \\
\hline
\end{tabular}

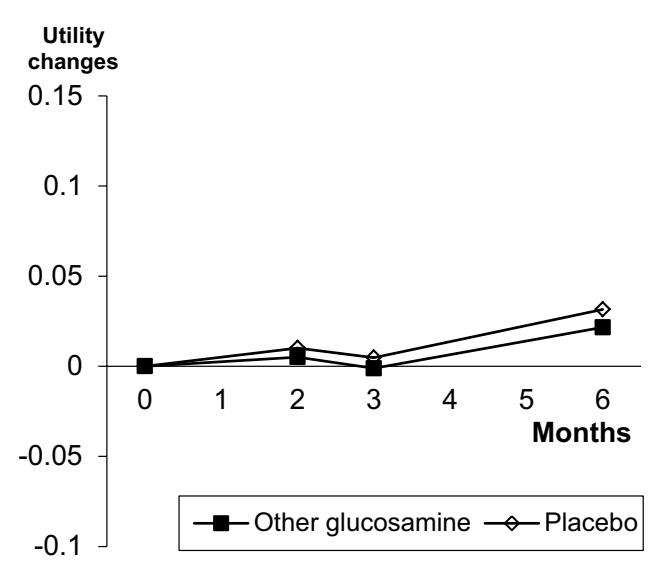

Fig. 2 Health utility score evolution using data from longer studies at all time points among studies that used pCGS or other forms of glucosamine

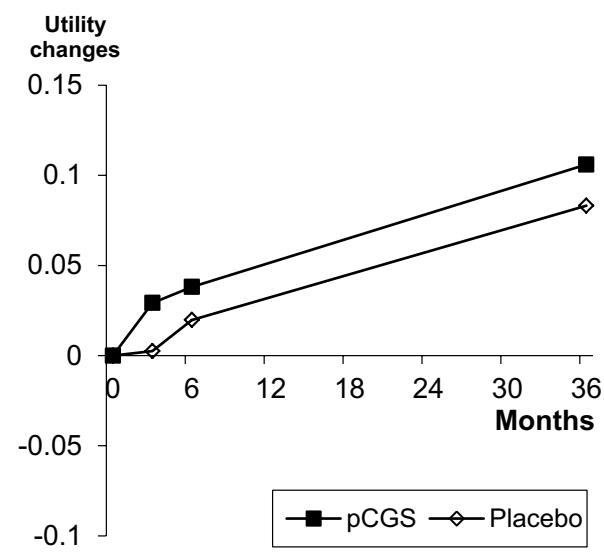

Table 4 Incremental cost-effectiveness ratio results of studies that used pCGS-sensitivity analyses

\begin{tabular}{lccc}
\hline & At 3 months & At 6 months & At 36 months \\
\hline QALY change pCGS & 0.00365489 & 0.01207722 & 0.19225352 \\
QALY change placebo & 0.00031524 & 0.00310523 & 0.13181955 \\
Median cost pCGS & 139.83 & 279.66 & 1677.96 \\
Median ICER & $41,869.6$ & $31,170.3$ & $27,765.1$ \\
Lowest cost pCGS & 115.05 & 230.1 & 1380.6 \\
Lowest ICER & $34,449.7143$ & $25,646.468$ & $22,844.7678$ \\
Higesth cost pCGS & 155.76 & 311.52 & 1869.12 \\
Highest ICER & $46,639.6132$ & $34,721.3721$ & $30,928.301$ \\
\hline
\end{tabular}

\section{Discussion}

In this study, we developed a model that could simulate individual patient data based on aggregated data found in published scientific papers. Indeed, most of the economic analyses require an access to individual patient data, which is unfortunately very difficult. Our model simulated all data needed to calculate the utility score (i.e., age, years since OA diagnosis, and WOMAC subscales). Using one validation study, our simulation model was able to provide summarised data similar to what could be found in the literature in term of utility score. It is also interesting to note that the
Table 5 Incremental costeffectiveness ratio results of studies that used other forms of glucosamine-sensitivity analyses

\begin{tabular}{llll}
\hline & At 2 months & At 3 months & At 6 months \\
\hline QALY change other glucosamine & 0.0004075 & 0.00056492 & 0.00311919 \\
QALY change placebo & 0.00080951 & 0.00140589 & 0.00595049 \\
Median cost other glucosamine & 33 & 59.4 & 118.8 \\
Median ICER & Placebo better & Placebo better & Placebo better \\
Lowest cost other glucosamine & 27 & 40.5 & 81 \\
Lowest ICER & Placebo better & Placebo better & Placebo better \\
Highest cost other glucosamine & 39.6 & 59.4 & 118.8 \\
Highest ICER & Placebo better & Placebo better & Placebo better \\
\hline
\end{tabular}


utility score calculated with the simulation model is within the range observed in patients with osteoarthritis [26].

With the individual patient data simulated from ten randomised controlled trials, we were able to calculate the ICER of different formulations of glucosamine. Our results showed that the use of pCGS only was cost-effective compared to placebo. To the best of our knowledge, only one clinical trial has been conducted, to assess, in post hoc analysis, the costeffectiveness of glucosamine. Using data form the GUIDE study, Scholtissen et al. showed that the use of pCGS was cost-effective compared to placebo but also to paracetamol [11]. However, one other study has assessed the health care utilisation following the use of pCGS [27]. In that particular 8-year observational study following a 3-year randomised controlled trial, the authors showed that patients formerly on pCGS had recurred with less symptomatic medications and the use of other health resources than did those from the placebo group during the last year of follow-up.

In this study, we also confirm the difference of (cost-) effectiveness among different glucosamine preparation. Indeed, our results highlight that pCGS, but not the other formulations of glucosamine is cost-effective. These results are supported by the recent meta-analysis of Eriksen et al. showing that the reduction of pain and the improvement in function was observed only with pCGS and not with the other types of glucosamine [9]. Glucosamine exists in different forms. However, all these products have not been characterised in the same way in terms of quality, pharmacokinetics and equivalence of human biological fluid levels with mechanistic data [28].

We have to acknowledge major limitations in our study. First, our simulation model has been validated using data from a single study only. Second, we did not have access to raw data to perform the ICER evaluation. Third, the cost evaluation was limited to the cost of the treatment and other costs have to be included to achieve a full health economics evaluation (e.g., the health care resources, the patient and family resources, the productivity costs and the others sectors resources). Fourth, some data needed to calculate the utility were unavailable in some publications, and we had to replace these data by data from the trial used to validate the model. Fifth, we had no direct assessment of the utility score and had to use the regression model developed and validated by Grootendorst et al. to obtain an estimation of the utility based on the WOMAC data [13], which is only one of the tools to assess quality of life in musculoskeletal health [29].

In conclusion, we confirm the superiority of pCGS over the other glucosamine formulations in terms of efficacy and cost-effectiveness. These results should be confirmed in other studies taking into account compliance to therapy, the other costs of OA management and a direct assessment of utility.

\section{Compliance with ethical standards}

Conflict of interest This study was supported by an unrestricted educational grant from MEDA. MEDA is the Marketing Authorisation Holder of crystalline glucosamine sulfate. The design, conduct and analysis of the study were under the sole responsibility of the Department of Public Health, Epidemiology and Health Economics of the University of Liège, without any intervention from third parties, including MEDA. Similarly, the preparation, writing, review and submission of the paper were under the sole responsibility of the authors. O. Bruyere reports grants from Biophytis, IBSA, MEDA, Servier, SMB, and Theramex. J-Y. Reginster reports grants from IBSA-Genevrier, Mylan, CNIEL, Radius Health, consulting fees from IBSA-Genevrier, Mylan, CNIEL, Radius Health, Pierre Fabre, fees for participation in review activities from IBSA-Genevrier, MYLAN, CNIEL, Radius Health, Teva, payment for lectures from AgNovos, CERIN, CNIEL, Dairy Research Council (DRC), Echolight, IBSA-Genevrier, Mylan, Pfizer Consumer Health, Teva, Theramex. Other have no potential conflicts of interest.

Research involving Human Participants and/or Animals Not applicable.

Informed consent Not applicable.

Open Access This article is distributed under the terms of the Creative Commons Attribution 4.0 International License (http://creativeco mmons.org/licenses/by/4.0/), which permits unrestricted use, distribution, and reproduction in any medium, provided you give appropriate credit to the original author(s) and the source, provide a link to the Creative Commons license, and indicate if changes were made.

\section{References}

1. Vina ER, Kwoh CK (2018) Epidemiology of osteoarthritis: literature update. Curr Opin Rheumatol 30:160-167. https://doi. org/10.1097/BOR.0000000000000479

2. Hochberg MC, Altman RD, April KT et al (2012) American College of Rheumatology 2012 recommendations for the use of nonpharmacologic and pharmacologic therapies in osteoarthritis of the hand, hip, and knee. Arthritis Care Res (Hoboken) 64:465-474

3. Zhang W, Nuki G, Moskowitz RW et al (2010) OARSI recommendations for the management of hip and knee osteoarthritis: part III: changes in evidence following systematic cumulative update of research published through January 2009. Osteoarthritis Cartilage 18:476-499. https://doi.org/10.1016/j.joca.2010.01.013

4. McAlindon TE, Bannuru RR, Sullivan MC et al (2014) OARSI guidelines for the non-surgical management of knee osteoarthritis. Osteoarthritis Cartilage 22:363-388. https://doi.org/10.1016/j. joca.2014.01.003

5. Bruyere O, Cooper C, Pelletier JP et al (2014) An algorithm recommendation for the management of knee osteoarthritis in Europe and internationally: a report from a task force of the European society for clinical and economic aspects of osteoporosis and osteoarthritis (ESCEO). Semin Arthritis Rheum 44:253-263. https:// doi.org/10.1016/j.semarthrit.2014.05.014

6. Nelson AE, Allen KD, Golightly YM et al (2014) A systematic review of recommendations and guidelines for the management of osteoarthritis: the chronic osteoarthritis management initiative of the U.S. bone and joint initiative. Semin Arthritis Rheum 43:701-712. https://doi.org/10.1016/j.semarthrit.2013.11.012

7. Reginster JY, Cooper C, Hochberg M et al (2015) Comments on the discordant recommendations for the use of symptomatic 
slow-acting drugs in knee osteoarthritis. Curr Med Res Opin 31:1041-1045. https://doi.org/10.1185/03007995.2015.1027183

8. Bruyere O, Cooper C, Al-Daghri NM et al (2018) Inappropriate claims from non-equivalent medications in osteoarthritis: a position paper endorsed by the European society for clinical and economic aspects of osteoporosis, osteoarthritis and musculoskeletal diseases (ESCEO). Aging Clin Exp Res 30:111-117. https://doi. org/10.1007/s40520-017-0861-1

9. Eriksen P, Bartels EM, Altman RD et al (2014) Risk of bias and brand explain the observed inconsistency in trials on glucosamine for symptomatic relief of osteoarthritis: a meta-analysis of placebo-controlled trials. Arthritis Care Res (Hoboken) 66:18441855. https://doi.org/10.1002/acr.22376

10. Hiligsmann M, Cooper C, Arden N et al (2013) Health economics in the field of osteoarthritis: an expert's consensus paper from the European society for clinical and economic aspects of osteoporosis and osteoarthritis (ESCEO). Semin Arthritis Rheum 43:303-313. https://doi.org/10.1016/j.semarthrit.2013.07.003

11. Scholtissen S, Bruyere O, Neuprez A et al (2010) Glucosamine sulphate in the treatment of knee osteoarthritis: cost-effectiveness comparison with paracetamol. Int J Clin Pract 64:756-762. https ://doi.org/10.1111/j.1742-1241.2010.02362.x

12. Black C, Clar C, Henderson R et al (2009) The clinical effectiveness of glucosamine and chondroitin supplements in slowing or arresting progression of osteoarthritis of the knee: a systematic review and economic evaluation. Health Technol Assess 13:1148. https://doi.org/10.3310/hta13520

13. Grootendorst P, Marshall D, Pericak D et al (2007) A model to estimate health utilities index mark 3 utility scores from WOMAC index scores in patients with osteoarthritis of the knee. J Rheumatol 34:534-542

14. Reginster JY, Deroisy R, Rovati LC et al (2001) Long-term effects of glucosamine sulphate on osteoarthritis progression: a randomised, placebo-controlled clinical trial. Lancet 357:251-256. https://doi.org/10.1016/S0140-6736(00)03610-2

15. Pavelka K, Gatterova J, Olejarova M et al (2002) Glucosamine sulfate use and delay of progression of knee osteoarthritis: a 3-year, randomized, placebo-controlled, double-blind study. Arch Intern Med 162:2113-2123

16. Herrero-Beaumont G, Ivorra JA, Del Carmen Trabado M et al (2007) Glucosamine sulfate in the treatment of knee osteoarthritis symptoms: a randomized, double-blind, placebo-controlled study using acetaminophen as a side comparator. Arthritis Rheum 56:555-567. https://doi.org/10.1002/art.22371

17. Giordano N, Fioravanti A, Papakostas P et al (2009) The efficacy and tolerability of glucosamine sulfate in the treatment of knee osteoarthritis: a randomized, double-blind, placebo-controlled trial. Curr Ther Res Clin Exp 70:185-196. https://doi. org/10.1016/j.curtheres.2009.05.004

18. Houpt JB, McMillan R, Wein C et al (1999) Effect of glucosamine hydrochloride in the treatment of pain of osteoarthritis of the knee. J Rheumatol 26:2423-2430
19. McAlindon T, Formica M, LaValley M et al (2004) Effectiveness of glucosamine for symptoms of knee osteoarthritis: results from an internet-based randomized double-blind controlled trial. Am J Med 117:643-649. https://doi.org/10.1016/j.amjmed.2004.06.023

20. Cibere J, Kopec JA, Thorne A et al (2004) Randomized, double-blind, placebo-controlled glucosamine discontinuation trial in knee osteoarthritis. Arthritis Rheum 51:738-745. https://doi. org/10.1002/art.20697

21. Clegg DO, Reda DJ, Harris CL et al (2006) Glucosamine, chondroitin sulfate, and the two in combination for painful knee osteoarthritis. N Engl J Med 354:795-808. https://doi.org/10.1056/ NEJMoa052771

22. Frestedt JL, Walsh M, Kuskowski MA et al (2008) A natural mineral supplement provides relief from knee osteoarthritis symptoms: a randomized controlled pilot trial. Nutr J 7:9. https://doi. org/10.1186/1475-2891-7-9

23. Chopra A, Saluja M, Tillu G et al (2011) A randomized controlled exploratory evaluation of standardized ayurvedic formulations in symptomatic osteoarthritis knees: a government of India NMITLI project. Evid Based Complement Altern Med 2011:724291. https ://doi.org/10.1155/2011/724291

24. Glick H, Doshi JA, Sonnad SS et al (2015) Economic evaluation in clinical trials. Handbooks in health economic evaluation series, 2nd edn. Oxford University Press, Oxford

25. Chopra A, Saluja M, Tillu G et al (2012) Comparable efficacy of standardized Ayurveda formulation and hydroxychloroquine sulfate (HCQS) in the treatment of rheumatoid arthritis (RA): a randomized investigator-blind controlled study. Clin Rheumatol 31:259-269. https://doi.org/10.1007/s10067-011-1809-z

26. Ruchlin HS, Insinga RP (2008) A review of health-utility data for osteoarthritis: implications for clinical trial-based evaluation. Pharmacoeconomics 26:925-935

27. Bruyere O, Pavelka K, Rovati LC et al (2008) Total joint replacement after glucosamine sulphate treatment in knee osteoarthritis: results of a mean 8-year observation of patients from two previous 3-year, randomised, placebo-controlled trials. Osteoarthritis Cartilage 16:254-260. https://doi.org/10.1016/j.joca.2007.06.011

28. Reginster JL, Bruyere O, Cooper C (2018) Different glucosamine sulfate products generate different outcomes on osteoarthritis symptoms. Ann Rheum Dis 77:e39. https://doi.org/10.1136/annrh eumdis-2017-212251

29. Beaudart C, Biver E, Bruyere O et al (2018) Quality of life assessment in musculo-skeletal health. Aging Clin Exp Res 30:413-418. https://doi.org/10.1007/s40520-017-0794-8

Publisher's Note Springer Nature remains neutral with regard to jurisdictional claims in published maps and institutional affiliations. 